\title{
Wer braucht Bildungsserver? Eine Studie zu Bekanntheit und Nutzung des Schweizerischen Bildungsservers im Vergleich verschiedener Nutzergruppen mit unterschiedlicher Medienaffinität
}

\section{Dominik Petko und Nives Egger}

In einer Befragung von 2432 Nutzenden und Nicht-Nutzenden des Schweizerischen Bildungsservers zeigt sich, dass die Informationsangebote des Bildungsservers nicht die zentrale Rolle haben wie im Leistungsauftrag anvisiert. Genutzt werden stattdessen vor allem die Gratis-Kommunikationswerkzeuge, die das Bildungsportal in Form einer Lernplattform anbietet. Differenzielle Analysen zeigen zudem, dass insbesondere Lehrpersonen aus höheren Schulstufen, Personen aus der Bildungsverwaltung sowie Personen mit einer hohen Medienaffinität die Angebote signifikant häufiger nutzen. Die Ergebnisse können Anstoss zu einer Neubeurteilung der Angebote von Bildungsportalen geben. Künftig könnten sich diese von "Anbietern vertrauenswürdiger Information» zu "Anbietern vertrauenswürdiger Plattformen» wandeln.

\section{Bildungsserver - ein Zukunfts- oder ein Auslaufmodell?}

Bildungsportale sind Websites, auf denen bildungsrelevante Informationen, Links und Werkzeuge zentral gesammelt und für interessierte Personen zur Verfügung gestellt werden (Jafari, 2003a; Kos, Lehmann, Brenstein \& Holtsch, 2005). Im Unterschied zu Suchmaschinen oder automatisierten News-Aggregatoren werden Bildungsportale typischerweise redaktionell betreut. Dadurch gibt es eine Qualitätskontrolle durch autorisierte Fachpersonen, die sicherstellen soll, dass Informationen korrekt und verlinkte Inhalte vertrauenswürdig sind. In vielen Ländern werden Bildungsserver im öffentlichen Auftrag oder sogar durch Regierungsbehörden selbst betrieben, was ihren Anspruch auf Verbindlichkeit und Glaubwürdigkeit unterstreicht. Beispiele für solche Bildungsserver sind z.B. der Deutsche Bildungsserver (www.bildungsserver.de), der Schweizerische Bildungsserver (www.educa.ch), das Österreichische Bildungsportal (www.schule.at), das Schottische (www.educationscotland.gov.uk) sowie der Irische Bildungsserver (www.scoilnet.ie) oder die Seite des U.S. Department of Education (www.ed.gov). Viele Bildungsserver haben ihre Angebotspalette in 
den letzten Jahren stark erweitert (Jafari, 2003b; Panke, 2007). Sie bieten nicht mehr nur Überblicksdarstellungen, kommentierte Linklisten und Sammlungen von Unterrichtsmaterialien, sondern auch Personalisierungs- und Community-Funktionen, interaktive Online-Tools und teilweise sogar vergünstigte kommerzielle Dienstleistungen für Bildungsinstitutionen. Dabei ist noch sehr wenig darüber bekannt, wie diese neuen Möglichkeiten genutzt werden. Gleichzeitig ist heute fraglich, ob das Modell des Bildungsservers auch künftig noch Bestand haben kann. Es gibt verschiedene Trends, die aktuell sowohl für als auch gegen das Modell der Bildungsserver angeführt werden können.

- Steigende Informationsmenge: Die Menge der bildungsrelevanten digitalen Informationen steigt. Dies betrifft einerseits die Anzahl von Webseiten, die im Unterricht eingesetzt werden können und andererseits speziell für Bildungszwecke produzierte offene Bildungsressourcen. Mit steigender Menge von Inhalten auf Bildungsservern werden diese potenziell ebenso unübersichtlich wie die Ergebnisse von Suchmaschinen. Auch die veralteten Links und Informationen auszusortieren, wird für redaktionell betriebene Bildungsserver $a b$ einer bestimmten Menge von Links und Informationen immer schwieriger. Der Schweizerische Bildungsserver besass bereits im Jahr 2010 schon mehr als 240‘000 HTML-Seiten (Petko, Graber \& Burton, 2010).

- Verbesserte Suchmaschinen: Die Algorithmen von Suchmaschinen werden immer treffsicherer. Wenn bildungsinteressierte Personen über Suchmaschinen sogar schneller zur gewünschten Information kommen als über die Katalogisierung und Verlinkung auf Bildungsportalen, dann stellt das deren Sinn in Frage. Gleichzeitig könnte es aber auch so sein, dass das eher zum Vorteil von Bildungsservern ist, da auch ihre Seiten über Suchmaschinen besser erschlossen. Schon heute ist es so, dass die Mehrheit der Zugriffe auf Inhalte von Bildungsservern über Suchmaschinen wie Google erfolgt (Böhm, 2011; Petko, Knüsel, Cantieni, Wespi \& Burton, 2011).

- Spezialisierte Portale: Neben umfassenden Bildungsservern, die typischerweise den Anspruch haben, alle möglichen bildungsrelevanten Informationen an einer zentralen Webadresse zu sammeln, existieren mittlerweile auch viele spezialisierte Portale, die sich auf einen Typus von Information beschränken. So stellt sich beispielsweise die Frage, wie umfassend Überblicksinformationen zum Bildungswesen noch auf Bildungsservern dargestellt werden müssen, wenn solche Informationen auch auf Wikipedia $\mathrm{zu}$ finden sind. Weiter gibt es lokale Bildungsportale, Unterrichtsmedienportale (z.B. www. zum.de) und vielfältige thematisch orientierte Portale (z.B. www.lehreronline.de). Bildungsserver könnten von dieser Entwicklung aber auch profitieren, wenn die Zersplitterung in Teilportale zu unübersichtlich wird oder sie selbst zu Betreibern von spezialisierten Portalen werden. Mit der Vielzahl von Bildungsportalen wird schon die Auswahl eines passenden Portals zu einer Herausforderung (Burke, 2005). 
- Open Educational Resources: Bildungsserver stehen angesichts des Trends zu offenen Bildungsressourcen («Open Educational Resources», vgl. Atkins, Brown \& Hammond 2007; Mruck, Mey, Schön, Idensen \& Purgathofer, 2013) vor der Frage, ob sie künftig in diesem Bereich grössere Verantwortung übernehmen wollen, sei es mit einer bevorzugten Verlinkung, eines Hosting in Repositorien, in der Qualitätskontrolle oder sogar in der Erstellung. Gleichzeitig könnte ein besonderer Fokus auf diese freien Inhalte ein weiteres Unterscheidungsmerkmal gegenüber kommerziellen Plattformen darstellen.

- Social Media: Bildungsinteressierte organisieren sich über Social Media Plattformen selbst. User werden auf Plattformen wie Facebook oder Twitter selbst zu Autor(inn)en und zu Redaktor(inn)en. Starre und hierarchische Verschlagwortungen werden abgelöst durch dynamische Folksonomies. Interessengemeinschaften formen sich dynamisch und betreiben ein eigenes dezentrales Informationsmanagement mittels Meldungen, Weiterleitungen und Bewertungen. Bildungsserver können von dieser Entwicklung jedoch unter Umständen auch profitieren, wenn es ihnen gelingt, diese Dynamik für die eigenen Inhalte nutzbar zu machen (z.B. Kerres \& Heinen, 2014).

- Medienaffinität und Informationskompetenz der Nutzenden: Wurden Bildungsportale früher teilweise noch als eine Hilfestellung für wenig interneterfahrene Personen gesehen, um wichtige Informationen gesammelt auf einer Adresse zu finden, so sind heute vermutlich mehr Personen in der Lage, sich die Informationen auch ohne die Hilfe von Bildungsservern selbst zusammenzusuchen. Jedoch könnte auch argumentiert werden, dass auch die Bildungsserver von einem stärker informationskompetenten Publikum profitieren. Obwohl erst wenige Studien zur Nutzung von Bildungsservern vorliegen, ist über förderliche und hinderliche Faktoren für die Nutzung von digitalen Medien im Unterricht vieles bekannt. Dabei zeigt sich, dass neben dem Zugang zudigitalen Technologien insbesondere Kompetenzen und Überzeugungen entscheidend sind (Ertmer \& Ottenbreit-Leftwich, 2010; Davies \& West, 2014). Solche personalen Faktoren können auch zu einem übergeordneten Konstrukt der Medienaffinität («tech-savvyness»; Borko, Whitcomb \& Liston, 2009; Schrum, Shelley \& Miller, 2008) zusammengefasst werden.

Angesichts solcher Entwicklungstendenzen, deren Auswirkungen nicht eindeutig abzuschätzen sind, lassen sich bei Bildungsservern heute vor allem drei unterschiedliche Strategien einer Neuausrichtung beobachten. Während einige sich auf ihre Rolle als redaktionell betreutes Informationsportal konzentrieren und ihre Aufgabe vor allem als Bildungsjournalismus verstehen (z.B. www.lehrer-online. de), erstellen andere praktisch keine eigenen Inhalte und setzen ausschliesslich auf Verlinkung (z.B. www.bildungsserver.de). Eine andere Strategie besteht darin, das Angebot eng mit weiterführender Beratung und Dienstleistung zu verknüpfen (z.B. www.learnline.schulministerium.nrw.de). Wieder andere konzentrieren sich vor allem auf das Bereitstellen technologischer Werkzeuge 
und Support (z.B. www.switch.ch). Daneben existieren weiterhin aber auch Bildungsserver, die alle genannten Bereiche integriert anbieten, darunter der Schweizerische Bildungsserver (www.educa.ch). Um solche Ausrichtungen des Angebots besser beurteilen zu können, stellt sich ganz grundsätzlich die Frage, welche Angebote von Bildungsservern heute überhaupt genutzt werden und von wem. Verschiedene Studien zeigen, dass die Nutzung von Bildungsportalen keine Selbstverständlichkeit ist, sondern stark von der Einfachheit der Nutzung und dem erlebten Nutzen abhängen (Mahmud, Ismail, Rahman, Kamarudin \& Ruslan, 2012; Pynoo et al., 2012). Deshalb ist es wichtig zu klären, welche Angebote in diesem Zusammenhang für Nutzende mit mehr oder weniger Medienaffinität attraktiv sind. Dass pädagogische Ressourcen für Lehrpersonen und ihre Unterrichtsentwicklung eine zentrale Bedeutung besitzen, ist heute unbestritten (Abramovich \& Schunn, 2012; Ball \& Cohen, 1996). Eine bessere Kenntnis der Nutzung von Bildungsservern kann eine wesentliche Grundlage dafür sein, Bildungsserver in einer sich wandelnden Online-Kultur weiterzuentwickeln und ihre Angebote als nützliche pädagogische Ressourcen zu etablieren.

\section{Der Schweizerische Bildungsserver}

Mit dem Schweizerischen Bildungsserver (www.educa.ch) wurde seit dem Jahr 2001 eine umfangreiche Informations- und Serviceplattform in allen vier Landessprachen (Deutsch, Französisch, Italienisch, Romanisch) sowie in Englisch entwickelt. Der Bildungsserver wird durch ein spezialisiertes Institut im offiziellen Auftrag der Schweizerischen Kantone und des Bundes betrieben. Er hat damit einen offiziellen Charakter für die ganze Schweiz, jedoch kommunizieren die Kantone daneben auch noch auf eigenen kantonalen oder regionalen Webseiten. Insgesamt enthält das Angebot des Schweizerischen Bildungsservers mehr als 240`000 HTML-Seiten sowie diverse interaktive Tools. Die Informationsinhalte sollen einen umfassenden Überblick über Themen des Bildungswesens vermitteln und Links auf relevante Ressourcen anbieten. Mit educanet2 (www.educanet2.ch) betreibt der Schweizerische Bildungsserver zudem ein auf die Bedürfnisse von Schulen angepasstes Learning Management System (LMS), das von Schweizer Bildungsinstitutionen gratis genutzt werden kann (Petko, 2010). Neben diesen freien Angeboten waren am Schweizerischen Bildungsserver zum Zeitpunkt der Studie verschiedene assoziierte Portale angegliedert, z.B. educashop oder educahelp, bei denen es sich um kommerzielle Dienstleistungen mit vergünstigten Angeboten für Schulen handelt. Der Leistungsauftrag des Schweizerischen Bildungsservers definiert drei primäre Zielgruppen des Angebots (Schweizerische Eidgenossenschaft \& Schweizerische Konferenz der kantonalen Erziehungsdirektoren, 2009): 
1) Akteure des Unterrichts, insbesondere Lehrpersonen aber auch Schülerinnen und Schüler

2) Unterstützende Personen aus dem lokalen Unterrichtsumfeld, insbes. Schulleitende

3) Personen aus dem steuernden und koordinierenden Unterrichtsumfeld

Insbesondere für Lehrpersonen versucht der Schweizerische Bildungsserver Online-Ressourcen zur Verfügung zu stellen, die sie in ihrer täglichen Arbeit unterstützen. Mit seiner äusserst breiten Angebotspalette kann der Schweizerische Bildungsserver nicht nur als gutes Beispiel dafür dienen, was alles möglich ist, sondern eine Untersuchung der Nutzung dieses Angebotes kann zutage fördern, welche Bereich des Angebotes den Zielgruppen besonders entsprechen.

\section{Fragestellungen}

Aus den bisherigen Ausführungen lassen sich die folgenden drei Forschungsfragen ableiten:

- Welche Angebote und Funktionen des Bildungsportals sind bei den Zielgruppen bekannt und welche werden häufig genutzt?

- Gibt es einen Unterschied in den Nutzungshäufigkeiten zwischen Lehrpersonen unterschiedlicher Schulstufen sowie zwischen Lehrpersonen und anderen professionellen Akteuren des Bildungswesens?

- Beeinflusst die Medienaffinität der Nutzenden deren Nutzung, einerseits bezüglich verschiedener Teile der Angebotspalette andererseits innerhalb bestimmter Nutzergruppen?

\section{Methoden}

Stichprobe und Datenerhebung

Im Auftrag der Schweizerischen Konferenz der kantonalen Erziehungsdirektoren (EDK) und des Staatssekretariats für Bildung, Forschung und Innovation (SBFI) wurde zwischen Anfang April und Mitte Mai 2011 eine gross angelegte Online-Umfrage zum Schweizerischen Bildungsserver durchgeführt. Die Befragung richtete sich sowohl an Nutzer/-innen als auch an Nicht-Nutzer/innen des Schweizerischen Bildungsservers. Sie erfolgte zwischen Anfangs April bis Mitte Mai 2011 mithilfe eines teilstandardisierten Online-Fragebogens auf Basis der Open Source Software LimeSurvey (www.limesurvey. org). Die Einladung zur Umfrage wurde über alle kantonalen Ämter für Volksschulen an Lehrpersonen und weitere Bildungsinteressierte verteilt. Links zur Online-Befragung wurden zudem über die Homepage und die Mailinglisten des 
Schweizerischen Bildungsservers zugänglich gemacht. Der Vorteil dieser Art der offenen Stichprobenziehung ist, möglichst breite Rückmeldungen zu erhalten. Der Nachteil liegt darin, dass keine genauen Angaben zu Repräsentativität und Rücklaufquote möglich sind. An der Befragung beteiligten sich 2432 Bildungsinteressierte aus allen Sprachregionen der Schweiz. Davon sind 52 Prozent männlich und 45 Prozent weiblich, 3 Prozent machten keine Angaben dazu. Die überwiegende Mehrheit der Fragebögen wurde in der Schweiz ausgefüllt, nur 64 Antwortende stammen aus dem Ausland, davon 16 aus Frankreich, 13 aus Deutschland, 8 aus Italien und 8 aus Liechtenstein. 50 Prozent der Fragebögen wurden in Deutsch, 47 Prozent in Französisch, 3 Prozent in Italienisch ausgefüllt. 7 Personen bearbeiteten den Fragebogen in Rätoromanisch. Das mittlere Alter der befragten Personen betrug $M=42$ Jahre bei einer Standardabweichung von $s=12$.

Bei den antwortenden Personen handelte es sich in sechs Prozent der Fälle um Lehrpersonen von Kindergarten/Unterstufe, bei 20 Prozent der Primarschule, bei 22 Prozent der Sekundarstufe I, bei 13 Prozent der Sekundarstufe II (Gymnasium und Berufsbildung) und bei sechs Prozent um Dozierende an Hochschulen. Kleine Anteile betreffen fünf Prozent Lehrpersonen für integrative Förderung, acht Prozent Schulleitende und vier Prozent Mitglieder von Bildungsverwaltungen. Eine weitere grössere Kategorie bilden die Anderen mit 16 Prozent. In diese Kategorie fallen alle Befragten, die keine unterrichtliche Tätigkeit ausüben oder nicht im Bereich des Bildungswesens berufstätig sind. Dies sind u.a. Schüler/-innen, Lehrlinge, Student/-innen, Weiterbildungsteilnehmende, Wissenschaftler, Personen aus Unternehmen oder Eltern.

In den standardisierten Teilen des Fragebogens wurden die Bekanntheit und Nutzungshäufigkeit der Angebote des Schweizerischen Bildungsservers mithilfe von vierpoligen Likertskalen beurteilt. Im Hinblick auf die Merkmale der Nutzenden wurden zudem wenige zusätzliche fünfpolige Fragen zur allgemeinen Häufigkeit der eigenen Internetnutzung, zu eigenen Fähigkeiten und Freude im Umgang mit dem Internet vorgelegt. Diese Antworten wurden vor weiteren Analysen entlang der untersuchten Hauptaspekte zu Maximalindizes bei ordinalskalierten Variablen bzw. Mittelwertsindizes bei intervallskalierten Variablen zusammengefasst. Alle Skalen besitzen mindestens akzeptable Reliabilitätswerte mit Cronbach's $\alpha \geq .70$. Der vollständige Datensatz dieser Befragung ist über den FORS data service mit der Projektnummer 11255 öffentlich verfügbar (http://forsdata.unil.ch).

\section{Datenauswertung}

Die vorliegenden Auswertungen stellen vertiefende Analysen dar, die über die Befunde des allgemeinen Schlussberichts (Petko, Knüsel, Cantieni, Wespi \& Burton, 2011) hinausgehen. Alle quantitativen Auswertungen wurden mittels R 3.02 und den Packages psych(), sjPlot(), Hmisc() sowie PMCMR() durchgeführt. Allen statistischen Analysen wurde ein Signifikanzniveau von $\mathrm{p}<.05$ zugrunde gelegt. Da die meisten Variablen nur eine Ordinalskalierung 
aufweisen, die Verteilung der Antworten selten normalverteilt und zudem die Vergleichsgruppen unterschiedlich gross sind, werden nonparametrische statistische Verfahren auf Basis von Rangsummenvergleichen eingesetzt Zur Prüfung von Unterschieden der Nutzungsfrequenz bei unterschiedlichen Nutzergruppen werden Kruskal-Wallis-Tests eingesetzt, für post-hoc Analysen signifikanter Ergebnisse dienen Nemenyi-Tests, die eine bessere Teststärke aufweisen als paarweise Vergleiche mit Wilcoxon-Tests mit Bonferroni-korrigiertem Signifikanzniveau (Pohlert, 2014). Diese Verfahren beruhen auf rangbasierten Verfahren, die statistisch sehr robust aber gleichzeitig auch relativ konservativ sind. Deshalb werden zusätzlich Effektstärken für signifikante Unterschiede berechnet. Dafür können die $\chi^{2}$-Werte der paarweisen post-hoc Tests zu r Koeffizienten transformiert werden (Ellis, 2010, p. 28). Gemäss Cohen (1988; 1992) kann $a b r>.10$ von einem kleinen, $a b r>.30$ von einem mittleren und $\mathrm{ab}>.50$ von einem grossen Effekt gesprochen werden. Die Prüfung korrelativer Zusammenhänge zwischen Medienaffinität und Stufen der Nutzung des Bildungsservers erfolgte mittels Spearman's rho Rangkorrelationskoeffizienten.

\section{Ergebnisse}

\section{Kenntnis und Nutzung der Angebote des Schweizerischen $B i$ ldungsservers}

Eine deskriptive Auswertung der Antworten zeigte zunächst, dass weniger als zwei Drittel der befragten Personen die Angebote des Schweizerischen Bildungsservers kennen und nur eine Minderheit sie häufig nutzen (Tabelle 1).

Tabelle 1. Kenntnis und Nutzung der einzelnen Angebote des Schweizerischen Bildungservers $(N=2433)$

\begin{tabular}{lcccc}
\hline & $\begin{array}{c}\text { kenne ich } \\
\text { nicht }\end{array}$ & $\begin{array}{c}\text { kenne ich, } \\
\text { nutze ich } \\
\text { aber nicht }\end{array}$ & $\begin{array}{c}\text { nutze ich } \\
\text { selten }\end{array}$ & $\begin{array}{c}\text { nutze ich } \\
\text { häufig }\end{array}$ \\
\hline 1. Überblicksinformationen und Links (V8) & $38 \%$ & $24 \%$ & $32 \%$ & $7 \%$ \\
2. Unterrichtsmaterialien zum Download (V9) & $39 \%$ & $26 \%$ & $28 \%$ & $7 \%$ \\
3. News und Agenda (V10) & $53 \%$ & $25 \%$ & $14 \%$ & $8 \%$ \\
4. Datenbank für Weiterbildungskurse (V18) & $65 \%$ & $25 \%$ & $9 \%$ & $1 \%$ \\
5. Online-Stellenbörse für Lehrpersonen (V12) & $55 \%$ & $31 \%$ & $9 \%$ & $5 \%$ \\
6. Online-Shop für Software und Hardware (V13) & $66 \%$ & $23 \%$ & $9 \%$ & $2 \%$ \\
7. Angebot für Schulverwaltungssoftware (V14) & $81 \%$ & $16 \%$ & $2 \%$ & $1 \%$ \\
8. Linux-Betriebssystem für Schulen (V15) & $83 \%$ & $15 \%$ & $2 \%$ & $1 \%$ \\
9. Fachtagungen für Lehrpersonen (V16) & $62 \%$ & $26 \%$ & $8 \%$ & $4 \%$ \\
10. Technische Beratung (V17) & $77 \%$ & $20 \%$ & $2 \%$ & $0 \%$ \\
11. Kommunikationsplattform educanet2 (V11) & $22 \%$ & $25 \%$ & $22 \%$ & $31 \%$ \\
\hline
\end{tabular}


Für eine prägnantere Verdichtung der Ergebnisse im Hinblick auf die erste Forschungsfrage wurden die Items zur Kenntnis und Nutzungshäufigkeit des Schweizerischen Bildungsservers in drei Kategorien zusammengefasst, die sich in dieser Gliederung auch im Leistungskatalog des Schweizerischen Bildungsservers finden (Schweizerische Eidgenossenschaft \& Schweizerische Konferenz der kantonalen Erziehungsdirektoren, 2009):

- Redaktionell aufgearbeitete Informationsangebote, die auf den Webseiten des Bildungsservers kostenlos abgerufen werden können (Angebote 1-5 der Tabelle 1: Cronbachs $\alpha=.78$ ),

- Erweiterte Services, die über die Webseiten des Bildungsservers gebucht werden können, die jedoch für Nutzende kostenpflichtig sind (Angebote 6-10 der Tabelle 1: Cronbachs $\alpha=.79$ )

- Die Lernplattform des Schweizerischen Bildungsservers, www.educanet2.ch, die eine breite Palette kostenfreier Kommunikationstools in sich integriert und deren Nutzung für schweizerische Bildungsinstitutionen kostenfrei ist (Einzelvariable 11 der Tabelle 1). Die Lernplattformeducanet2 umfasst eine E-Mail-Umgebung, eine Dateiablage und zahlreiche weitere Werkzeuge wie Foren, Chats, Wikis und Blogs. .

Für die weitere Arbeit mit diesen Kategorien wurden Maximalindizes der Antworten aus diesen Angebotsbereichen erstellt, d.h. es wurde in jedem Bereich das am besten bekannte bzw. am intensivsten genutzte Angebot aus diesem Bereich als Kennwert zugrunde gelegt. Eine deskriptive Auswertung dieser Indizes findet sich in Tabelle 2.

Tabelle 2. Kenntnis und Nutzung der Angebotsbereiche des Schweizerischen Bildungsservers $(N=2433)$

\begin{tabular}{lcccc}
\hline & $\begin{array}{c}\text { kenne ich } \\
\text { nicht }\end{array}$ & $\begin{array}{c}\text { kenne ich, } \\
\text { nutze ich } \\
\text { aber nicht }\end{array}$ & $\begin{array}{c}\text { nutze ich } \\
\text { selten }\end{array}$ & $\begin{array}{c}\text { nutze ich } \\
\text { häufig }\end{array}$ \\
\hline 1. Kostenlose Informationsanagebote auf educa.ch & $23 \%$ & $20 \%$ & $36 \%$ & $20 \%$ \\
2. Kostenpflichtige Services bei educa.ch & $48 \%$ & $31 \%$ & $14 \%$ & $7 \%$ \\
3. Kommunikationsplattform educanet2.ch & $22 \%$ & $25 \%$ & $22 \%$ & $31 \%$ \\
\hline
\end{tabular}

Diese verdichteten Darstellungen der Tabelle 2 zeigen, dass es signifikante Unterschiede zwischen in der Bekanntheit und Nutzung der verschiedenen Angebotsbereiche des Bildungsservers bestehen $\left(\chi^{2}(2)=809.52, \mathrm{p}<.001\right)$. In post hoc Tests wird zudem deutlich, dass es in der Summe nicht die redaktionell erstellten Informationsangebote sind, die die höchsten Bekanntheits- bzw. Nutzungszahlen aufweisen, sondern dass die vom Bildungsserver über eine Lernplattform angebotenen Kommunikationstools insgesamt noch leicht häufiger genutzt werden $(\mathrm{r}=.05, \mathrm{p}<.05)$. Kostenpflichtige Services fallen in Bekanntheit und 
Nutzung deutlich ab, sowohl im Vergleich mit der Lernplattform ( $\mathrm{r}=.51$, $\mathrm{p}<.001)$ als auch im Vergleich zu den kostenlosen Informationsangeboten $(\mathrm{r}=.45, \mathrm{p}<.001)$.

\section{Kenntnis und Nutzungshäufigkeit des Bildungsservers nach Berufsgruppen}

Der Schweizerische Bildungsserver verfügt über äusserst heterogene Zielgruppen und bisher ist erst wenig darüber bekannt, ob der Bildungsserver diese Gruppen gleichermassen erreicht. Um die Vergleichsgruppen nicht zu heterogen oder zu klein werden zu lassen, konzentrieren sich die folgenden Analysen auf professionelle Unterrichtsakteure, d.h. Lehrpersonen vom Kindergarten bis zur Sekundarstufe II ( $\mathrm{n}=1515)$ sowie schulische Sonder- und Sozialpädagoginnen ( $\mathrm{n}=148)$, Schulleitende $(\mathrm{n}=185)$ und Personen aus Schulbehörden und Bildungspolitik $(\mathrm{n}=134)$. Bei Angabe von mehreren Funktionen auf unterschiedlichen Ebenen war jeweils die höchste Ebene massgeblich für die Zuordnung. Bei der grossen Gruppe der Lehrpersonen ist es zudem möglich und sinnvoll, unterschiedliche Schulstufen und Sonderfunktionen zu unterscheiden, die sich bezüglich ihrer Ausstattung und Nutzung digitaler Medien ebenso wie bezüglich ihrer allgemeinen Rahmenbedingungen deutlich unterscheiden (Petko, 2012). Bei der Kenntnis und der Nutzung der Bereiche des Schweizerischen Bildungsservers zeigen sich deutliche Unterschiede zwischen diesen verschiedenen Gruppen, d.h. sowohl bei den Informationsangeboten $\left(\chi^{2}(7)=65.13, p<.001\right)$ wie bei den kostenpflichtigen Services $\left(\chi^{2}(7)=99.53, \mathrm{p}<.001\right)$ und den Angeboten der Kommunikationsplattform $\left(\chi^{2}(7)=28.06, \mathrm{p}<.001\right)$.

Tabelle 3 zeigt die Verteilung der Antworten nach Berufsgruppen in Bezug auf die Informationsangebote des Bildungsservers.

Tabelle 3. Kenntnis und Nutzung der redaktionell erstellten und kostenlosen Informationsangebote des Schweizerischen Bildungsservers bei professionellen Akteuren des Bildungssystems ( $N=1982)$

\begin{tabular}{lccccc}
\hline & $\mathrm{n}$ & $\begin{array}{c}\text { kenne ich } \\
\text { nicht }\end{array}$ & $\begin{array}{c}\text { kenne ich, } \\
\text { nutze ich } \\
\text { aber nicht }\end{array}$ & $\begin{array}{c}\text { nutze ich } \\
\text { selten }\end{array}$ & $\begin{array}{c}\text { nutze ich } \\
\text { häufig }\end{array}$ \\
\hline 1. Lehrperson Kindergarten & 136 & $29 \%$ & $18 \%$ & $38 \%$ & $15 \%$ \\
2. Lehrperson Primarstufe & 496 & $23 \%$ & $19 \%$ & $38 \%$ & $21 \%$ \\
3. Lehrperson Sekundarstufe I & 532 & $23 \%$ & $22 \%$ & $37 \%$ & $18 \%$ \\
4. Lehrperson Sek II Beruf & 98 & $27 \%$ & $28 \%$ & $28 \%$ & $18 \%$ \\
5. Lehrperson Sek II Allgemein & 253 & $25 \%$ & $29 \%$ & $30 \%$ & $16 \%$ \\
6. Sozial-/Sonderpädagogik & 148 & $19 \%$ & $16 \%$ & $43 \%$ & $22 \%$ \\
7. Schulleitung & 185 & $11 \%$ & $19 \%$ & $44 \%$ & $26 \%$ \\
8. Bildungsbehörden & 134 & $7 \%$ & $12 \%$ & $49 \%$ & $32 \%$ \\
\hline
\end{tabular}

Post hoc: $7>1^{* *}, 3^{* *}, 4^{*}, 5^{* * *} ; 8>1^{* * *}, 2^{* * *}, 3^{* * *}, 4^{* * *}, 5^{* * *} ; * p<.05$, ** $p<.01$, *** $p<.001$ 
Die Post-hoc-Tests bezüglich der Nutzung von Informationsressourcen zeigen, dass keine signifikanten Unterschiede zwischen den verschiedenen Gruppen von Lehrpersonen bestehen. Hingegen nutzen Schulleitende die Informationsressourcen signifikant häufiger als fast alle Gruppen von Lehrpersonen, mit Ausnahme der Primarlehrpersonen. Auch Personen aus der Bildungsverwaltung zeigen in den paarweisen Gruppenvergleichen eine intensivere Nutzung der Informationsressourcen als Lehrpersonen aller Stufen. Zwischen Schulleitenden und Personen aus der Bildungsverwaltung finden sich keine signifikanten Unterschiede in der Nutzungshäufigkeit der Informationsressourcen des Schweizerischen Bildungsservers. Ein ähnliches Muster zeigt sich bei der Nutzung der kostenpflichtigen Services des Bildungsservers (Tabelle 4).

Tabelle 4. Kenntnis und Nutzung der kostenpflichtigen Dienstleistungen des Schweizerischen Bildungsservers bei professionellen Akteuren des Bildungssystems $(N=1982)$

\begin{tabular}{lccccc}
\hline & n & $\begin{array}{c}\text { kenne } \\
\text { ich nicht }\end{array}$ & $\begin{array}{c}\text { ich, nutze } \\
\text { ich aber } \\
\text { nicht }\end{array}$ & $\begin{array}{c}\text { nutze ich } \\
\text { selten }\end{array}$ & $\begin{array}{c}\text { nutze ich } \\
\text { häufig }\end{array}$ \\
\hline 1. Lehrperson Kindergarten & 136 & $61 \%$ & $29 \%$ & $8 \%$ & $2 \%$ \\
2. Lehrperson Primarstufe & 496 & $51 \%$ & $32 \%$ & $12 \%$ & $5 \%$ \\
3. Lehrperson Sekundarstufe I & 532 & $45 \%$ & $32 \%$ & $16 \%$ & $7 \%$ \\
4. Lehrperson Sek II Beruf & 98 & $47 \%$ & $31 \%$ & $8 \%$ & $14 \%$ \\
5. Lehrperson Sek II Allgemein & 253 & $51 \%$ & $28 \%$ & $14 \%$ & $7 \%$ \\
6. Sozial-/Sonderpädagogik & 148 & $45 \%$ & $35 \%$ & $16 \%$ & $4 \%$ \\
7. Schulleitung & 185 & $27 \%$ & $43 \%$ & $21 \%$ & $10 \%$ \\
8. Bildungsbehörden & 134 & $22 \%$ & $32 \%$ & $28 \%$ & $17 \%$ \\
\hline
\end{tabular}

Post hoc: $3>1^{*} ; 7>1^{* * *}, 2^{* * *}, 3^{* * *}, 5^{* * *}, 6^{*} ; 8>1^{* * *}, 2^{* * *}, 3^{* * *}, 4^{* * *}, 5^{* * *}, 6^{* * *} ;{ }^{*} p<.05,{ }^{* *} p<$ $.01,{ }^{* * *} p<.001$

Die Post-hoc Tests bezüglich der Nutzung kostenpflichtiger Services zeigen, dass sowohl Angehörige der Bildungsverwaltung diese Angebote häufiger kennen und nutzen als Lehrpersonen aller Schulstufen. Auch Schulleitungspersonen zeigen hier signifikant höhere Nutzungszahlen als Lehrpersonen aller Schulstufen, mit Ausnahme Berufsschullehrpersonen. Einen kleinen, jedoch signifikanten Unterschied gibt es zudem zwischen Lehrpersonen der Sek I und Kindergartenlehrpersonen. In Bezug auf die Nutzung der Kommunikationsplattform finden sich diese Unterschiede hingegen nicht (Tabelle 5). 
Tabelle 5. Kenntnis und Nutzung der Kommunikationsplattform des Schweizerischen Bildungsservers bei professionellen Akteuren des Bildungssystems $(N=1982)$

\begin{tabular}{lccccc}
\hline & n & $\begin{array}{c}\text { kenne } \\
\text { kenne } \\
\text { ich nicht }\end{array}$ & $\begin{array}{c}\text { ich, nutze } \\
\text { ich aber } \\
\text { nicht }\end{array}$ & $\begin{array}{c}\text { nutze ich } \\
\text { selten }\end{array}$ & $\begin{array}{c}\text { nutze ich } \\
\text { häufig }\end{array}$ \\
\hline 1. Lehrperson Kindergarten & 136 & $32 \%$ & $22 \%$ & $25 \%$ & $21 \%$ \\
2. Lehrperson Primarstufe & 496 & $22 \%$ & $24 \%$ & $19 \%$ & $35 \%$ \\
3. Lehrperson Sekundarstufe I & 532 & $27 \%$ & $21 \%$ & $23 \%$ & $39 \%$ \\
4. Lehrperson Sek II Beruf & 98 & $27 \%$ & $24 \%$ & $19 \%$ & $31 \%$ \\
5. Lehrperson Sek II Allgemein & 253 & $15 \%$ & $30 \%$ & $25 \%$ & $30 \%$ \\
6. Sozial-/Sonderpädagogik & 148 & $26 \%$ & $21 \%$ & $24 \%$ & $29 \%$ \\
7. Schulleitung & 185 & $14 \%$ & $27 \%$ & $23 \%$ & $36 \%$ \\
8. Bildungsbehörden & 134 & $10 \%$ & $31 \%$ & $25 \%$ & $33 \%$ \\
\hline
\end{tabular}

Post hoc: $1<2^{* *}, 3^{* * *}, 5^{*}, 7^{* * *}, 8^{* *} ;{ }^{*} p<.05,{ }^{* *} p<.01,{ }^{* * *} p<.001$

Bei der Nutzung der Kommunikationsplattform sind es lediglich die Kindergartenlehrpersonen, die diese Plattform signifikant weniger gut kennen und weniger häufig nutzen als Lehrpersonen aller anderen Stufen mit Ausnahme der Gymnasiallehrpersonen. Kindergartenlehrpersonen nutzen die Plattform ausserdem ebenfalls signifikant weniger häufig als Schulleitungspersonen und Mitglieder der Bildungsbehörden. Es gibt jedoch keine signifikanten Unterschiede zwischen Lehrpersonen anderer Stufen sowie zwischen Lehrpersonen und Schulleitungsmitgliedern oder Angehörigen von Bildungsbehörden.

Auch wenn die Lehrpersonen verschiedener Stufen zu einer Gruppe zusammengelegt werden, sind die Unterschiede zwischen den Nutzergruppen hoch signifikant, sowohl bezüglich der Informationsangebote $\left(\chi^{2}(2)=51.31, \mathrm{p}<.001\right)$ als auch der kostenpflichtigen Services $\left(\chi^{2}(2)=82.65, \mathrm{p}<.001\right)$. Die Effektstärken der Differenzen sind jedoch relativ klein. Die Nutzung der Informationsangebote durch Lehrpersonen fällt in den post hoc Tests zusammengenommen leicht tiefer aus als die der Schulleitungsmitglieder $(\mathrm{r}=.11, \mathrm{p}<.001)$ und der Personen aus dem steuernden Umfeld ( $\mathrm{r}=.17, \mathrm{p}<.001)$. Gleiches gilt für den Bereich der kostenpflichtigen Services, die Lehrpersonen zusammengenommen ebenfalls weniger kennen und nutzen als Schulleitungsmitglieder $(\mathrm{r}=.15, \mathrm{p}<.001)$ oder als Vertreter der Bildungsbehörden ( $r=.20, \mathrm{p}<.001)$. Keine Unterschiede zwischen den Gruppen zeigen sich hingegen bei der Kenntnis und Nutzung der Kommunikationswerkzeuge der Lernplattform $\left(\chi^{2}(2)=3.03\right.$, n.s.) die damit nicht nur am häufigsten genutzt werden, sondern deren Nutzung über die verschiedenen Zielgruppen hinweg am homogensten ausfällt. 


\section{Kenntnis und Nutzungshäufigkeit des Bildungsservers nach Medienaffinität}

Die dritte Frage der vorliegenden Untersuchung richtet sich darauf, ob Personen, die eine höhere «durchschnittliche Internetnutzung pro Woche», bessere «Kenntnisse im Umgang mit digitalen Medien» sowie mehr "Freude am Umgang mit digitalen Medien» aufweisen, die Angebote des Bildungsservers häufiger nutzen als solche, die geringere Ausprägungen dieser Merkmale aufweisen. Diese drei Variablen wurden zunächst z-standardisiert und dann zu einem wiederum z-standardisierten Indexwert «Medienaffinität» zusammengefasst (3 Items, Cronbach's $\alpha=.76$ ).

Bei einer rangbasierten Korrelation zwischen Medienaffinität und Bekanntheit bzw. Nutzung der einzelnen Angebote des Bildungsservers zeigt sich, dass hohe Medienaffinität mit einer höheren Nutzung praktisch aller Angebote des Bildungsservers einhergeht (Tabelle 6). Eine hohe Medienaffinität scheint insbesondere für die Nutzung der Fachtagungen und des Online-Shops entscheidend zu sein. Hingegen scheint dieser Zusammenhang bei den Datenbanken für Weiterbildungs- oder Jobangebote nicht ganz so eng zu sein.

Tabelle 6. Rangkorrelationen zwischen digitaler Medienaffinität der Befragten und Kenntnis/Nutzung der verschiedenen Angebote des Schweizerischen Bildungsservers $(N=1981)$

\begin{tabular}{lc}
\hline & $\rho$ \\
\hline 1. Überblicksinformationen und Links (V8) & $.27^{* * *}$ \\
2. Unterrichtsmaterialien zum Download (V9) & $.23^{* * *}$ \\
3. News und Agenda (V10) & $.28^{* * *}$ \\
4. Datenbank für Weiterbildungskurse (V18) & $.10^{* * *}$ \\
5. Online-Stellenbörse für Lehrpersonen (V12) & $.16^{* * *}$ \\
6. Online-Shop für Software und Hardware (V13) & $.34^{* * *}$ \\
7. Angebot für Schulverwaltungssoftware (V14) & $.16^{* * *}$ \\
8. Linux-Betriebssystem für Schulen (V15) & $.27^{* * *}$ \\
9. Fachtagungen für Lehrpersonen (V16) & $.32^{* * *}$ \\
10. Technische Beratung (V17) & $.23^{* * *}$ \\
11. Lernplattform educanet2 (V11) & $.25^{* * *}$ \\
\hline
\end{tabular}

Spearman's $\rho$,Signifikanzniveau ${ }^{* * *} \mathrm{p}<.001$

Fasst man diese Angebotspalette wieder zu den drei Hauptleistungsbereichen zusammen, dann zeigt sich für jeden dieser Bereiche eine deutliche Korrelation zwischen ihrer Nutzung und der digitalen Medienaffinität der Nutzenden. Bei den kostenlosen Informationsangeboten zeigt sich insgesamt ein kleiner positiver jedoch hoch signifikanter Zusammenhang (Spearman's $\rho=.26, \mathrm{p}<$ .001 ), ebenso bei der Kommunikationsplattform (Spearman's $\rho=.25, \mathrm{p}<.001$ ). Ein grösserer Zusammenhang besteht hier bei der Nutzung kostenpflichtiger 
Services (Spearman's $\rho=.37, \mathrm{p}<.001$ ), die noch einmal im stärkeren Masse Personen mit höherer Medienaffinität bekannt zu sein scheinen.

Ein Vergleich der Medienaffinität (5-stufige Likertskala 1 = sehr geringe Medienaffinität $-5=\operatorname{sehr}$ hohe Medienaffinität) der untersuchten Berufsgruppen zeigt zunächst, dass hier durchaus signifikante Unterschiede bestehen $\left(\chi^{2}(7)=133.11, \mathrm{p}<.001\right)$. Gemäss post-hoc Tests kann dabei die Tendenz festgestellt werden, dass Lehrpersonen der jeweils höheren Schulstufen eine höhere Medienaffinität zeigen als Lehrpersonen aus tieferen Schulstufen. Kindergartenlehrpersonen haben eine tiefere Medienaffinität als sämtliche anderen Berufsgruppen, mit Ausnahme der Sonder- und Sozialpädagog(inn)en. Gleiches gilt für die Primarlehrpersonen, die dabei jedoch eine signifikant höhere Medienaffinität aufweisen als die Kindergartenlehrpersonen. Lehrpersonen der Sekundarstufe I haben zwar eine höhere Medienaffinität als Lehrpersonen niedrigerer Stufen, aber auch eine geringere als Lehrpersonen der allgemeinbildenden Sekundarstufe II. Die Lehrpersonen für Sekundarstufe II (Beruf und Allgemein) bilden die medienaffinste Gruppe unter den Lehrpersonen und bewegen sich dabei auf einem vergleichbaren Niveau wie die Schulleitungen. Die Gruppe mit der höchsten Medienaffinität setzen sich aus Personen der Bildungssteuerung, d.h. Schul- und Bildungsbehörden zusammen (Tabelle 7).

\section{Tabelle 7. Z-standardisierte Mittelwerte und Standardabweichungen der} Medienaffinität im Vergleich der Nutzergruppen

\begin{tabular}{lcc}
\hline Nutzergruppen & $\mathrm{n}$ & $\begin{array}{c}\text { Medienaffinität } \\
\mathrm{M}(\mathrm{s})\end{array}$ \\
\hline 1. Lehrperson Kindergarten & 136 & $-.52(.91)$ \\
2. Lehrperson Primarstufe & 495 & $-.25(.99)$ \\
3. Lehrperson Sekundarstufe I & 531 & $-.04(1.01)$ \\
4. Lehrperson Sek II Beruf & 97 & $.23(.98)$ \\
5. Lehrperson Sek II Allgemein & 253 & $.21(.90)$ \\
6. Sozial-/Sonderpädagogik & 148 & $-.42(1.11)$ \\
7. Schulleitung & 185 & $.19(.85)$ \\
8. Bildungsbehörden & 134 & $.42(.89)$ \\
\hline
\end{tabular}

Post-hoc: $1<2^{* * *}, 3^{* * *}, 4^{* * *}, 5^{* * *}, 7^{* * *}, 8^{* * *} ; 2<3^{*}, 4^{* * *}, 5^{* * *}, 7^{* * *}, 8^{* * *} ; 3<5^{*}, 8^{* * *} ; 3>6^{* *} ; 4>6^{* * *} ; 5$ $>6^{* * *} ; 6<7^{* * *}, 8^{* * *} ;{ }^{*} p<.05,{ }^{* *} p<.01,{ }^{* * *} p<.001$

Im Anschluss an diese Analysen stellt sich letztlich die Frage, ob die Medienaffinität die Unterschiede zwischen den Berufsgruppen bei der Nutzung des Bildungsservers erklären kann. Das für diese Zwecke geeignete Verfahren, die univariate Kovarianzanalyse, konnte aufgrund fehlender Normalverteilung und Varianzhomogenität sowie heterogenen Regressionskoeffizienten nicht durchgeführt werden. Aus diesem Grund wurde als alternatives Verfahren eine einfache Rangkorrelation zwischen der Nutzung der einzelnen Angebotsbereiche und der Medienaffinität, aufgeschlüsselt nach Berufsgruppen gewählt (Tabelle 8). 
Tabelle 8. Korrelation von Medienaffinität und Nutzung des Schweizerischen Bildungsservers nach Nutzergruppen

\begin{tabular}{|c|c|c|c|c|}
\hline $\begin{array}{l}\text { Medienaffinität korreliert mit } \\
\text { der Nutzung der }\end{array}$ & & $\begin{array}{l}\text { Kostenlosen } \\
\text { Informa- } \\
\text { tions- } \\
\text { angebote }\end{array}$ & $\begin{array}{l}\text { Kosten- } \\
\text { pflichtigern } \\
\text { Services }\end{array}$ & $\begin{array}{l}\text { Kommuni- } \\
\text { kations- } \\
\text { plattform }\end{array}$ \\
\hline Nach Nutzergruppen & $n$ & $\rho$ & $\rho$ & $\rho$ \\
\hline 1. Lehrperson Kindergarten & 136 & .10 & .06 & $.31^{* * *}$ \\
\hline 2. Lehrperson Primarstufe & 495 & $.28^{* * *}$ & $.32^{* * *}$ & $.17^{* * *}$ \\
\hline 3. Lehrperson Sekundarstufe I & 531 & $.26^{* * *}$ & $.29^{* * *}$ & $.31^{* * *}$ \\
\hline 4. Lehrperson Sek II Beruf & 97 & .20 & $.35^{* * *}$ & $.34^{* * *}$ \\
\hline 5. Lehrperson Sek II Allgemein & 253 & $.23^{* * *}$ & $.30^{* * *}$ & $.14^{*}$ \\
\hline 6. Sozial-/Sonderpädagogik & 148 & $.43^{* * *}$ & $.51^{* * *}$ & $.28^{* * *}$ \\
\hline 7. Schulleitung & 185 & .12 & $.20^{* *}$ & $.20^{* *}$ \\
\hline 8. Bildungsbehörden & 134 & $.39^{* * *}$ & $.49^{* * *}$ & $.32^{* * *}$ \\
\hline
\end{tabular}

Spearman's rho,Signifikanzniveau ${ }^{*} \mathrm{p}<.05,{ }^{* *} \mathrm{p}<.01,{ }^{* * *} \mathrm{p}<.001$

Die Ergebnisse zeigen, dass die Medienaffinität nicht bei allen Nutzergruppen und allen Angeboten gleichermassen eine Rolle spielt. Ausserdem zeigt sich kein einfach zu interpretierendes Muster. Bei den Angehörigen von Schulleitungen scheint es für eine intensivere Nutzung der Angebote des Bildungsservers weniger auf Medienaffinität anzukommen als bei den Vertretern von Bildungsbehörden. Bei Lehrpersonen zeigen sich nahezu durchgängig positive und moderate Korrelationen, ausser bei den Kindergartenlehrpersonen, bei denen sich möglicherweise aber auch Bodeneffekte zeigen. Bei Fachpersonen aus dem Bereich Sozialund Sonderpädagogik scheint Medienaffinität hingegen besonders entscheidend mit einer stärkeren Nutzung des Bildungsservers einherzugehen.

Zwar kann Hypothese 3 damit tendenziell dahingehend bestätigt werden, dass eine höhere Medienaffinität mit einer intensiveren Nutzung der Angebote des Bildungsservers einhergeht. Im Detail zeigen sich jedoch Spezifitäten innerhalb der Nutzergruppen, die eine Generalisierung dieser Aussage relativieren.

\section{Schlussfolgerungen und Diskussion}

In dieser Studie wurde mit einer grossen Befragung von mehr als 2400 Personen untersucht, welche Angebote und Funktionen des Schweizerischen Bildungsservers bekannt sind und mit welcher Intensität diese Angebote genutzt werden. Darüber hinaus wurde der Frage nachgegangen, welche Berufsgruppen im Bildungswesen dieses Bildungsportal nutzt und welche Rolle die Medienaffinität der Nutzer und Nutzerinnen dabei spielt. Der Schweizerische Bildungsserver ist hier exemplarisch, da er zum Zeitpunkt der Untersuchung ein besonders breites 
Angebot verschiedener Informations-, Kommunikations- und Dienstleistungsservices umfasste.

Die Studie zeigt, dass die für Nutzende frei verfügbaren, redaktionell erstellten Informationsangebote des Bildungsservers sowohl einzeln als auch in ihrer Summe weniger bekannt sind und seltener genutzt werden als die kostenlose Lernplattform, die vom Schweizerischen Bildungsserver ebenfalls angeboten wird. Dieser Befund hinterfragt das eingangs skizzierte Muster, nach dem eine Informationsplattform das eigentliche Kernangebot von Bildungsservern darstellt, für das üblicherweise auch die grössten Ressourcen aufgewendet werden. Kostenpflichtige Services, die vom Bildungsserver ebenfalls angeboten werden, sind allerdings noch einmal deutlich weniger bekannt als diese beiden Angebotsbereiche und werden nur von vergleichsweise wenigen Personen genutzt. Diese Befragungsergebnisse decken sich mit Logfile-Analysen zur Nutzung des Schweizerischen Bildungsservers aus dem Untersuchungszeitraum, aus denen bekannt ist, dass neben der Hauptseite insbesondere der Bereich der Unterrichtsressourcen zum Download auf dem Bildungsserver den meisten Besuche erhält (Petko, Knüsel, Cantieni, Wespi \& Burton, 2011). Auch die Lernplattform educanet2 wird jedoch nicht in ihrem vollen Funktionsumfang genutzt. Aus einer früheren Untersuchung unter Einschluss von Logfile-Analysen ist bekannt, dass hier jedoch fast ausschliesslich die Funktionen E-Mail und Dateiablage genutzt werden (Petko, 2010).Ausserdem konnte die Studie verschiedene Unterschiede zwischen Nutzergruppen identifizieren. Obwohl sich die Angebote des Bildungsserver gemäss eigenem Anspruch vor allem an Lehrpersonen richten, zeigt die Studie, dass gerade diese Berufsgruppe, die kostenlosen Informationsangebote und die kostenpflichtigen Services weniger gut kennt und nutzt als Schulleitende und Personen aus dem steuernden Umfeld. Nur die Lernplattform scheint bei allen untersuchten Nutzergruppen, mit Ausnahme von Kindergartenlehrpersonen, gleichermassen bekannt zu sein.

Als bedeutsamer Faktor für die Nutzung des Schweizerischen Bildungsservers hat sich in der Studie zudem die Medienaffinität erwiesen. Personen, die digitale Medien regelmässig nutzen, sich damit nach eigener Einschätzung gut auskennen und sich auch gerne damit beschäftigen, kennen und nutzen auch die Angebote des Schweizerischen Bildungsservers häufiger als Personen mit geringerer Medienaffinität. Besonders deutlich ausgeprägt ist dieser Zusammenhang bei den kostenpflichtigen Services. Obwohl Lehrpersonen höherer Schulstufen sowie Schulleitende und Angehörige aus dem steuernden Umfeld tendenziell eine höhere Medienaffinität aufweisen, lassen sich die Unterschiede zwischen den Berufsgruppen nur teilweise mit der Medienaffinität erklären.

Die Studie weist mehrere methodische Einschränkungen auf. Mit der Untersuchung lassen sich nur Aussagen über die Quantität und nicht über die Qualität der Nutzung von Bildungsservern machen. Alle Ergebnisse der Nutzerinnen und Nutzer basieren zudem auf selbstberichteten Daten aus diesem Grund sind diese möglicherweise nicht frei von subjektiver Verzerrung, wobei die allerdings 
erwähnten Logfile-Analysen zumindest die Daten zu Nutzungsfrequenzen der verschiedenen Angebote zu stützen scheinen. Die Befragungen waren zudem sehr kurz, so dass nur ausgewählte Aspekte abgefragt wurden und dadurch z.B. umfangreiche Befragungen zu den theoretischen Facetten von Technologieakzeptanz (z.B. wie bei Pynoo et al., 2012) nicht möglich waren. Die Ergebnisse beziehen sich zudem nur auf den Schweizerischen Bildungsserver und es ist unklar, ob sich diese Ergebnisse auch auf andere Bildungsserver übertragen lassen. Auch die Repräsentativität der Stichprobe ist nicht zweifelsfrei erwiesen, da anzunehmen ist, dass vor allem interessierte Personen sich zur Teilnahme an der Befragung bereit erklärt haben. Dass allerdings sogar diese Personen die Informations- und Serviceangebote des Bildungsservers kaum kennen und selten nutzen, zeigt, dass Bildungsserver möglicherweise nicht die Breitenwirkung erzielen, die sie dem Anspruch nach haben (Schweizerische Eidgenossenschaft \& Schweizerische Konferenz der kantonalen Erziehungsdirektoren, 2009). Stattdessen zeigt sich die höchste Bekanntheit und Nutzung bei der Lernplattform educanet2. Über die Gründe lässt sich nur spekulieren. Sie können entweder darin liegen, dass die Nutzung von educanet2 an manchen Schulen und in manchen Kantonen zumindest als Mail- und Dokumentenaustauschplattform obligatorisch ist (Petko, 2010). Möglicherweise zeigt sich hier jedoch auch ein grundsätzlicherer Trend, den der Schweizerische Bildungsserver zum Anlasse nehmen könnte um sein Angebot zu überdenken. Statt die Rolle des «trusted information providers» in den Vordergrund zu stellen, könnten Bildungsserver in Zukunft möglicherweise auch verstärkt die Rolle eines «trusted platform providers» übernehmen. Schulen hätten hier für Anwendungen wie E-Mail, Sozialen Netzwerke und Dateiablage die Möglichkeit, auf Angebote des öffentlichen Dienstes zurückzugreifen statt auf solche privater Firmen mit teilweise undurchsichtigen Geschäftsbedingungen und Datenschutzbestimmungen. Für eine Klärung solcher Fragen müssten allerdings künftige Studien die Rolle von Bildungsservern in der erweiterten Informationsökologie von Bildungsakteuren untersuchen. Es ist heute noch weitgehend unklar, wie z.B. Lehrpersonen sich informieren, mit Kollegen vernetzen und auf ihren Unterricht vorbereiten. Digitale Medien und Bildungsserver sind hier nur ein Baustein in einem grösseren Puzzle.

\section{Literatur}

Abramovich, S. \& Schunn, C. (2012). Studying teacher selection of resources in an ultralarge scale interactive system: Does metadata guide the way? Computers \& Education, 58, 551-559.

Atkins, D. E., Brown, J. S. \& Hammond, A. L. (2007). A review of the open educational resources (OER) movement: Achievements, challenges, and new opportunities. Creative common. Retrieved 28.08.2015 from: http://www.hewlett.org/uploads/files/ReviewoftheOERMovement.pdf

Ball, D. L. \& Cohen, D. K. (1996). Reform by the book: What is: or might be: the role of curriculum materials in teacher learning and instructional reform? Educational researcher, $25,6-14$. 
S. 542: Böhm, P. (2011). Ermittlung von Nutzungsweisen auf dem Deutschen Bildungsserver mittels Webanalyse-Verfahren. Zugriff am 28.08.2015 unter http://www.pedocs.de/ volltexte/2011/3800/pdf/boehm2010_nutzungsweisen_dbs_masterarbeit_D_A.pdf

Borko, H., Whitcomb, J. \& Liston, D. (2009). Wicked Problems and Other Thoughts on Issues of Technology and Teacher Learning. Journal of Teacher Education, 60(1), 3-7.

Burke, J. (2005). Educational Web Portals: Guidelines for Selection and Use. A Guide To Help School District Administrators Assess the Quality, Usefulness and Reliability of Commercially Provided Educational Web Portals Designed for Schools. Retrieved DATE month day, year, from http://www.sreb.org/programs/EdTech/pubs/PDF/Web_Portals.pdf

Cohen, J. (1988). Statistical power analysis for the behavioral sciences (2nd ed.). Hillsdale, NJ: Erlbaum.

Cohen, J. (1992). Quantitative methods in psychology - a power primer. Psychological Bulletin, 112(1), 155-159.

Davies, R. \& West, R. (2014). Technology Integration in Schools. In J. M. Spector, M. D. Merrill, J. Elen \& M. J. Bishop (Ed.), Handbook of Research on Educational Communications and Technology (pp. 841-853). Springer New York.

Ellis, P. D. (2010). The Essential Guide to Effect Sizes. Statistical Power, Meta-Analysis, and Interpretation of Research Results. Cambridge: Cambridge University Press.

Ertmer, P. A. \& Ottenbreit-Leftwich, A. (2010). Teacher Technology Change. How knowledge, beliefs, and culture intersect. Journal of Research on Technology in Education, 42(3), 255-284.

Jafari, A. (2003a). Educational Portal White Paper. In A. Jafari \& M. Sheehan (Ed.), Designing Portals: Opportunities and Challenges (pp. 270-290). Hershey, PA: IRM Press.

Jafari, A. (2003b). The Next Generation of Internet Portals. In A. Jafari \& M. Sheehan (Ed.), Designing Portals: Opportunities and Challenges (p. 89.100). Hershey, PA: IRM Press.

Kerres, M. \& Heinen, R. (2014). Open Educational Resources und schulisches Lernen: Das Zusammenwirken von Plattformen für Lernressourcen in informationell offenen Ökosystemen.In P. Missomelius, W. Sützl, T. Hug, P. Grell \& R. Kammerl (Hrsg.), Medien - Wissen - Bildung: Freie Bildungsmedien und Digitale Archive / Media, Knowledge \& Education: Open Educational Resources and Digital Archives. Innsbruck: Innsbruck University Press.

Kos, O., Lehmann, R., Brenstein, E. \& Holtsch, D. (2005). Bildungsportale - Wegweiser im Netz: Gestaltung - Nutzung - Evaluation. Frankfurt am Main: Peter Lang.

Mahmud, R., Ismail, M. A. H., Rahman, F. A., Kamarudin, N. \& Ruslan, A. R. (2012). Teachers' Readiness in Utilizing Educational Portal Resources in Teaching and Learning. Procedia-Social and Behavioral Sciences, 64, 484-491.

Mruck, K., Mey, G., Schön, S., Idensen, H. \& Purgathofer, P. (2013). Offene Lehr-und Forschungsressourcen. Open Access und Open Educational Resources. In M. Ebner \& S. Schön (Ed.), L3T: Lehrbuch für Lehren und Lernen mit Technologien (http://l3t.eu), Version 2013. Graz: TU Graz.

Panke, S. (2007). Ingredients of Educational Portals as Infrastructures for Informal Learning Activities. In T. Bastiaens \& S. Carliner (Ed.), Proceedings of World Conference on E-Learning in Corporate, Government, Healthcare, and Higher Education 2007 (pp. 1203-1212). Chesapeake, VA: AACE.

Petko, D. (2010). Die Lernplattform educanet2 in der Schweiz. In D. Petko (Ed.), Lernplattformen in Schulen. Ansätze für E-Learning und Blended Learning in Präsenzklassen (pp. 29-42). Wiesbaden: VS-Verlag. doi:10.1007/978-3-531-92299-7_2

Petko, D. (2012). Hemmende und förderliche Faktoren des Einsatzes digitaler Medien im Unterricht: Empirische Befunde und forschungsmethodische Probleme. In R. SchulzZander, B. Eickelmann, H. Moser, H. Niesyto, \& P. Grell (Ed.), Jahrbuch Medienpädagogik 9 (pp. 29-50). Wiesbaden: Springer. doi:10.1007/978-3-531-94219-3_3. 
Petko, D., Graber, M. \& Burton, S. (2010). Controlling Schweizerischer Bildungsserver. Bericht 2010. Goldau: Institut für Medien und Schule, PHZ Schwyz.

Petko, D., Knüsel, D., Cantieni, A., Wespi, B. \& Burton, S. (2011). Controlling Schweizerischer Bildungsserver. Bericht 2011. Goldau: Institut für Medien und Schule, PHZ Schwyz.

Pohlert, T. (2014). The Pairwise Multiple Comparison of Mean Ranks Package (PMCMR). Online unter http://cran.r-project.org/web/packages/PMCMR/vignettes/PMCMR.pdf.

Pynoo, B., Tondeur, J., Van Braak, J., Duyck, W., Sijnave, B. \& Duyck, P. (2012). Teachers' acceptance and use of an educational portal. Computers \& Education, 58, 1308-1317.

Schrum, L., Shelley, G. \& Miller, R. (2008). Understanding tech-savvy teachers: Identifying their characteristics, motivation, and challenges. International Journal of Technology in Teaching and Learning, 4(1), 1-20.

Schweizerische Eidgenossenschaft \& Schweizerische Konferenz der kantonalen Erziehungsdirektoren (2009). Leistungskatalog vom 19. Juni 2009 betreffend Betrieb des Schweizerischen Bildungsservers SBS. Anhang zum Vertrag betreffend Betrieb des Schweizerischen Bildungsservers SBS in der Periode 2009 - 2012. Bern : BBT/EDK.

Schlagworte: Bildungsserver, Open Educational Resources, ICT, Befragung

\section{Qui a besoin de serveurs éducatifs? Étude au sujet des groupes d'utilisateurs et de leurs profils respectifs}

\section{Résumé}

Cette recherche est partie de l'hypothèse que les informations fournies par le Serveur suisse de l'éducation tiennent un rôle central. Une enquête interrogeant 2432 utilisateurs et non utilisateurs de ce serveur montre qu'à l'inverse de cette supposition, les informations livrées par le serveur n'ont pas l'importance qu'on leur prête. À contrario, ce sont les outils de communication gratuits (plate-forme d'apprentissage) proposés par le portail de l'éducation qui sont utilisés. Des formes différentielles montrent que ce sont particulièrement les enseignants des degrés supérieurs, les cadres de l'éducation ainsi que les personnes habituées à utiliser ce type de média qui utilisent plus souvent - et de manière significative - les offres du Serveur suisse de l'éducation. Les résultats peuvent inciter à une réévaluation des prestations proposées pars les portails éducatifs. Dorénavant celles-ci pourraient, au lieu de s'adresser à de «prestataires d'informations dignes de confiance» s'adresser à des"prestataires de plates-formes dignes de confiance».

Mots-clé: Serveur de l'éducation, ressources éducatives libres, MITIC, enquête 


\section{A chi servono i portali didattici? Uno studio sulla diffusione e I'uso die portali didattici svizzeri da parte di diversi gruppi di utenti con profili mediatici diversi.}

Riassunto

Un sondaggio di 2432 utenti e non utenti dello Swiss Educational Server ha mostrato che le risorse informative gratuite del portale sono meno conosciute e usate di quanto ci si aspettasse. Al contrario, gli strumenti di comunicazione offerti dall'ambiente di apprendimento online del portale sono meglio conosciuti e più usati. Inoltre, analisi differenziali mostrano che gli insegnanti di settori scolastici più avanzati così come i decisori in ambito educativo hanno maggior probabilità di utilizzare il portale rispetto a docenti dei livelli inferiori. Inoltre, lo studio mostra che individui con più affinità nell'uso dei media hanno usato i servizi dello Swiss Educational Services significativamente più spesso. I risultati offrono un incentivo per rivalutare i servizi dei portali educativi. Nel futuro, i portali potrebbero presentarsi in maniera più marcata come «offerta di piattaforma affidabile» che come "offerta di materiali informativi affidabili».

Parole chiave: Server per l'educatione, Risorse didattiche aperte, ICT, sondaggio

\section{Who Needs Educational Portals? A Survey on Users and Usage}

Summary

A survey of 2432 users and non-users of the Swiss Educational Server showed that the portals' free information resources are not as well known and as widely used as expected. Instead, the communication tools offered by the portal's learning management system are better known and used more often. In addition, differential analyses show that teachers at higher levels of the educational system as well as education policy-makers are likelier to use the educational portal than teachers at lower levels. Moreover, it was shown that individuals with higher media affinity used the services offered by the Swiss Educational Services significantly more frequently. The findings provide an incentive for reevaluating the services of educational portals. In the future, portals could put greater emphasis on their role as "trusted platform providers» rather than as «trusted information providers».

Keywords: Educational portal, open educational resources, ICT, survey 


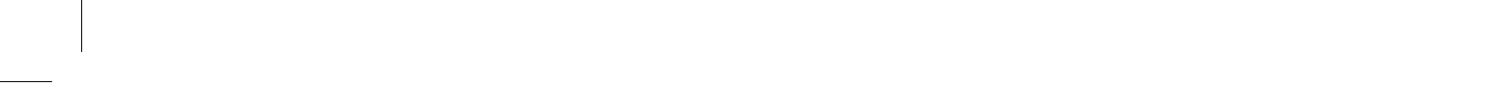

